\title{
Comprehensive, structurally-informed alignment and phylogeny of vertebrate biogenic amine receptors
}

Stephanie Spielman, Keerthana Kumar, Claus O. Wilke

Biogenic amine receptors play critical roles in regulating behavior and physiology, particularly within the central nervous system, in both vertebrates and invertebrates. Members of the G-protein coupled receptor (GPCR) family, these receptors interact with endogenous bioamine ligands, such as dopamine, serotonin, and epinephrine, and they are targeted by a wide array of pharmaceuticals. Despite these receptors' clear clinical and biological importance, their evolutionary history remains poorly characterized. In particular, the relationships among biogenic amine receptors and any specific evolutionary constraints acting within distinct receptor subtypes are largely unknown. To advance and facilitate studies in this receptor family, we have constructed a comprehensive, highquality sequence alignment of vertebrate biogenic amine receptors. In particular, we have integrated a traditional multiple sequence approach with robust structural domain predictions to ensure that alignment columns accurately capture the highly-conserved GPCR structural domains, and we demonstrate how ignoring structural information produces spurious inferences of homology. Using this alignment, we have further constructed a structurally-partitioned maximum-likelihood phylogeny, from which we deduce novel biogenic amine receptor relationships and uncover previously unrecognized lineage-specific receptor clades. Moreover, we find that roughly $1 \%$ of the 3039 sequences in our final alignment are either misannotated or unclassified, and we propose updated classifications for these receptors. We release our comprehensive alignment and its corresponding phylogeny as a resource for future research into the evolution and diversification of biogenic amine receptors. 


\title{
Comprehensive, structurally-informed alignment and phylogeny of vertebrate biogenic amine receptors
}

\author{
Stephanie J. Spielman ${ }^{1,2,3}$, Keerthana Kumar ${ }^{1,2,3}$, and Claus O. Wilke ${ }^{1,2,3}$ \\ ${ }^{1}$ Department of Integrative Biology, The University of Texas at Austin, Austin, U.S.A. \\ ${ }^{2}$ Institute of Cellular and Molecular Biology, The University of Texas at Austin, Austin, U.S.A. \\ ${ }^{3}$ Center for Computational Biology and Bioinformatics, The University of Texas at Austin, Austin, \\ U.S.A.
}

\begin{abstract}
Biogenic amine receptors play critical roles in regulating behavior and physiology, particularly within the central nervous system, in both vertebrates and invertebrates. Members of the G-protein coupled receptor (GPCR) family, these receptors interact with endogenous bioamine ligands, such as dopamine, serotonin, and epinephrine, and they are targeted by a wide array of pharmaceuticals. Despite these receptors' clear clinical and biological importance, their evolutionary history remains poorly characterized. In particular, the relationships among biogenic amine receptors and any specific evolutionary constraints acting within distinct receptor subtypes are largely unknown. To advance and facilitate studies in this receptor family, we have constructed a comprehensive sequence alignment of vertebrate biogenic amine receptors. In particular, we have integrated a traditional multiple sequence approach with robust structural domain predictions to ensure that alignment columns accurately capture the highly-conserved GPCR structural domains, and we demonstrate how ignoring structural information produces spurious inferences of homology. Using this alignment, we have further constructed a structurally-partitioned maximum-likelihood phylogeny, from which we deduce novel biogenic amine receptor relationships and uncover previously unrecognized lineage-specific receptor clades. Moreover, we find that roughly $1 \%$ of the 3039 sequences in our final alignment are either misannotated or unclassified, and we propose updated classifications for these receptors. We release our comprehensive alignment and its corresponding phylogeny as a resource for future research into the evolution and diversification of biogenic amine receptors.
\end{abstract}

Keywords: biogenic amine receptors, G-protein coupled receptors, multiple sequence alignment, phylogenetics

\section{INTRODUCTION}

Biogenic amines, such as the molecules serotonin and dopamine, play critical roles in virtually all Metazoans and exert significant influence on both behavior and physiology. In vertebrates, the biogenic amine receptor family, which includes dopamine (DRD), histamine (HRH), trace (TAAR), adrenergic (ADR), muscarinic cholinergic (mAChR), and most serotonin (5HTR) receptors, primarily mediates biogenic amine activity. Biogenic amine receptors belong to the broad family of $\mathrm{G}$ protein-coupled receptors (GPCRs), one of the largest and most diverse eukaryotic receptor families. Indeed, due to the extensive diversity of biological functions they direct and the ongoing expansion of their ligand repertoire, GPCRs are considered one of the most evolutionarily innovative and successful gene families (Bockaert and Pin, 1999; Lagerstrom and Schioth, 2008).

Biogenic amine receptors form a clade within the large Rhodopsin-like GPCR family (Fredriksson et al., 2003, Kakarala and Jamil, 2014), whose emergence likely accompanied that of the Opisthokont (Fungi and Metazoa) lineage (Krishnan et al. 2012). The Rhodopsin-like family expanded substantially in Metazoa, and the specific diversification of biogenic amine receptors has contributed significantly to central nervous system functioning (Callier et al. 2003; Nichols and Nichols 2008). Like all GPCRs, biogenic amine receptors have a characteristic, highly-conserved structure of seven transmembrane (TM) domains separated by three extracellular (ECL) and three intracellular (ICL) loops, and they propagate intracellular signaling through a G-protein-mediated pathway. Moreover, these receptors are prominent targets for a wide range of pharmaceuticals aimed to treat myriad diseases such as schizophrenia, migraines, hypertension, allergies and asthma, and stomach ulcers (Schoneberg et al., 2004; Evers et al., 2005; Mason et al., 2012).

In spite of these receptors' biological and clinical importance, studies on their evolution are limited and have predominantly focused on individual receptor subtypes, namely TAAR (Gloriam et al., 2005; Lindemann et al. 2005; Hashiguchi 
and Nishida, 2007), DRD (Callier et al., 2003; Yamamoto et al., 2013), and 5HTR (Anbazhagan et al. 2010). Moreover, many of these studies, and indeed studies on the general evolution of the Rhodopsin-like family, have examined very narrow species distributions, for instance specifically teleosts (Gloriam et al., 2005), primates (Anbazhagan et al., 2010), humans and mice (Vassilatis et al., 2003, Kakarala and Jamil, 2014), or even strictly humans (Fredriksson et al., 2003). Thus, virtually no studies have been conducted that account for the full breadth of vertebrate biogenic amine receptor sequences.

To gain a comprehensive understanding of this receptor family's evolution, a robust multiple sequence alignment (MSA) is needed. MSAs provide the foundation for nearly all comparative sequence analyses, and they are commonly used to locate conserved sequence motifs, identify functionally important residues, and investigate evolutionary histories. As constructing an MSA represents the first step in any sequence analysis, MSA errors are known to bias these downstream analyses (Ogden and Rosenberg, 2006, Wong et al., 2008; Jordan and Goldman, 2012). It is therefore crucial to ensure accuracy in MSAs to the extent possible.

For GPCR sequences, in particular, any MSA should recapitulate the canonical seven-TM structure, which a naive alignment of sequences cannot necessarily accomplish. Several varieties of MSA software platforms have been developed that incorporate structural information into the alignment algorithm by aligning sequences to a given protein crystal structure (Pei et al., 2008, O'Sullivan et al., 2004) or hidden Markov model (HMM) profile (Eddy, 1998, Chang et al., 2012, Hill and Deane, 2012). In fact, some programs, such as MP-T (Hill and Deane, 2012) and TM-Coffee (Chang et al., 2012), cater specifically to membrane proteins. However, all of these programs are extremely computationally-intensive and thus ill-suited for large-scale applications. Furthermore, many of these programs require the use of a single crystal structure or HMM profile to guide sequence alignment. While all GPCRs contain seven TM domains, different GPCR subfamilies, particularly the biogenic amine receptors, feature a wide variety of ICL and ECL sizes. For example, human HRH1 and DRD3 contain roughly 27 and 117 residues, respectively, in their ECL3 domains, and roughly 68 and 14 residues, respectively, in their ICL3 domains [as predicted by GPCRHMM (Wistrand et al., 2006)]. Thus, aligning diverse sequences using a single structure may not effectively capture the domain variability across biogenic amine receptor subtypes. Instead, a desirable alignment strategy would anchor all sequences by their conserved TM domains without inappropriately constraining the heterogeneous ECL and ICL domains.

To this end, we integrated a traditional progressive alignment approach with robust structural predictions to generate a structurally-informed, comprehensive (3039 sequences) MSA of vertebrate biogenic amine receptors, representing the most extensive such dataset to date. Through this strategy, we overcame the computational limitations imposed by explicitly structurally-aware MSA construction platforms while still incorporating structural information into MSA construction. We used our structurally-informed MSA to build a maximum likelihood (ML) phylogeny of vertebrate biogenic amine receptors, and we found that a partitioned phylogeny which separately considered TM and extramembrane (EM) domains dramatically improved phylogenetic fit relative to an unpartitioned phylogeny. Using this structurally-partitioned phylogeny, we were able to discern relationships among biogenic amine receptor subtypes with a far increased level of sensitivity relative to previous studies, as well as identify novel lineage-specific receptor clades and clarify NCBI annotations for over 30 sequences.

We present this vertebrate biogenic amine receptor MSA and its corresponding phylogeny as a resource for any group interested in studying the dynamic evolutionary processes and structural and/or functional constraints operating within this class of GPCRs. All data, including MSAs, phylogenies, and sequence descriptions, as well as all code used to generate these data, are freely available from https://github.com/s jspielman/amine_receptors. We expect that these data will prove useful for studying both the broad patterns governing biogenic amine receptor sequence evolution and the evolutionary trends specific to certain receptor subtypes. Further, our MSA should serve as a helpful resource in the ongoing development of homology models and pharmaceutical therapeutics targeting these receptors (Kristiansen, 2004, Ishiguro, 2004; Evers et al., 2005; Mason et al. 2012).

\section{METHODS AND MATERIALS}

\section{Sequence Collection and Processing}

We collected protein sequences using PSI-BLAST (Altschul et al., 1997), specifically from the RefSeq (v2.2.29+) database (Pruitt et al. 2013), for 42 distinct human biogenic amine protein sequences representing the full range of known such receptors in the human genome. To obtain distant yet well-supported orthologs, we ran each PSI-BLAST search for 5 iterations with an e-value cutoff of $10^{-20}$, a sequence identity threshold of $25 \%$, and a length difference of $\pm 50 \%$ relative to the seed sequence. After combining all sequences recovered from the individual PSI-BLAST searches, we discarded duplicate sequences, leaving a total of 4232 PSI-BLAST results. We then filtered this sequence set to remove sequences from non-vertebrate taxa, sequences annotated as low-quality, pseudogene, and/or partial, and sequences which contained more than $1 \%$ ambiguous residues (i.e. B, X, or Z). We additionally used the program GPCRHMM (Wistrand et al., 2006) to determine whether a given sequence was indeed a GPCR. We discarded sequences which had either a local or 
global GPCRHMM score less than 10, both extremely conservative thresholds. Thus, while it is possible that some true GPCRs were discarded, these stringent thresholds for both local and global scores provide high confidence that all retained sequences were indeed GPCRs. Together, these filters left a total of 3464 receptor sequences.

\section{Sequence Alignment and Phylogenetic Reconstruction}

Before aligning sequences, we used the program GPCRHMM (Wistrand et al.,2006) to assign each residue in all protein sequences to its respective structural domain (extracellular, transmembrane, or intracellular) using a 0.5 posterior probability cutoff. We then aligned and filtered sequences according to the strategy outlined in Figure 3 , which specifically employed MAFFT v7.149b using the default algorithm (Katoh and Standley, 2013).

All phylogenies were created using RAxML v8.1.1 (Stamatakis, 2014) using the LG (Le and Gascuel, 2008) amino acid exchangeability matrix with empirical amino acid frequencies $(+\mathrm{F})$ and the CAT model of site heterogeneity (Stamatakis, 2006), with the default 25 rate categories. For inferences incorporating structural partitions, we assigned each partition a unique evolutionary model using these settings, thus allowing each partition to have distinct equilibrium amino-acid frequency and rate distributions. Final parameter values for all phylogenetic inferences were optimized with the GAMMA model of heterogeneity. We performed 100 phylogenetic inferences for each parameterization to thoroughly search the tree space, and we used a likelihood ratio test (LRT) to compare the best resulting trees from each parameterization. We computed 200 bootstrapped trees using RAxML for each resulting phylogeny.

\section{RESULTS AND DISCUSSION}

\section{Constructing a structurally-informed MSA of biogenic amine receptors}

We collected all biogenic amine receptor sequences from the RefSeq database (Pruitt et al., 2013) using PSI-BLAST and removed all poor-quality sequences (see Methods for details). We then used the software GPCRHMM (Wistrand et al., 2006) to identify whether each sequence in our data set was indeed a GPCR. GPCRHMM uses a hidden Markov model approach to identify GPCRs from protein sequence data alone, and features an exceptionally low false positive rate $(\sim 1 \%)$ as well as a $15 \%$ increase in sensitivity relative to other similar structural prediction programs (Wistrand et al., 2006). We removed all sequences which GPCRHMM could not robustly classify as a GPCR, leaving a dataset of 3464 protein sequences.

In addition to identifying GPCR sequences, GPCRHMM can also predict transmembrane domain regions for Rhodopsinlike GPCRs with exceptional accuracy (Spielman and Wilke, 2013). As shown in Figure 1, GPCRHMM yields excellent domain predictions for resolved biogenic amine receptor crystal structures and thus serves as a robust proxy for more computationally-intensive structural predictors. We therefore used GPCRHMM to predict the structural domain (TM, extracellular, or intracellular) for each residue in these sequences.

To begin, we then aligned all 3464 protein sequences with MAFFT (Katoh and Standley, 2013), with default settings. Using GPCRHMM's domain predictions, we determined the consensus structural domain for each MSA column to assess how well the MSA recapitulated the overarching GPCR domain structure (Figure 2A). We found that, although we had already filtered out putatively non-GPCR sequences, several hundred sequences did not align according to the overarching domain structure. Many sequences were shifted out of structural frame, causing TM domains to inappropriately align with loop domains, or vice versa. Moreover, the mere presence of these misaligned sequences in the naive MSA introduced a substantial amount of gaps, often times within a single TM domain (notably TM1 and TM7, as seen in Figure 2A). While gaps are not inherently problematic in MSAs, the strong evolutionary pressures conserving GPCR structure should prevent large indel events from occurring within TM domains. Thus, many of the large gaps in this alignment were likely produced by the presence of confounding sequences in the dataset.

We therefore adopted an iterative strategy integrating progressive sequence alignment with the GPCRHMM structural predictions to systematically cull poorly-aligned sequences. As outlined in Figure 3 , we first aligned protein sequences with MAFFT (Katoh and Standley, 2013). Using the residue domain assignments computed with GPCRHMM, we determined the consensus domain for each column in this MSA. Next, we discarded all sequences for which $\geq 5 \%$ of residues did not correspond to their respective column's consensus domain. We realigned the remaining sequences with MAFFT and continued in this manner until no more sequences were discarded. Importantly, this strategy did not require any manual data filtering or visual data inspection, thus avoiding any confounding subjectivity in MSA processing. Ultimately, this strategy produced a final MSA which indeed captures the conserved GPCR domain structure, as depicted in Figure 23 . This structurally-informed MSA contains 3039 sequences broadly distributed across receptor subtypes (Table 1) and vertebrate taxa (Figure 4). We additionally noted that the structurally-informed MSA contains canonical Rhodopsin-family GPCR motifs, including the E/DRY motif at the boundary of TM3 and ICL2 (MSA columns 2351-3) and the NPxxY motif at the boundary of TM7 and the C-terminal loop (MSA columns 3411-5). 


\section{Structurally-aware MSA strongly improves phylogenetic fit}

Next, we used this structurally-informed MSA to infer a maximum likelihood (ML) phylogeny of vertebrate biogenic amine receptors in RAxML. Previous work has shown that combined structural and functional constraints impose differing selection pressures in TM vs. EM domains, in turn producing distinct amino-acid frequencies and evolutionary rates in each domain class (Tourasse and Li, 2000; Stevens and Arkin, 2001; Julenius and Pedersen, 2006; Oberai et al., 2009, Spielman and Wilke, 2013; Franzosa et al., 2013). As our MSA allowed us to confidently identify each MSA column as either TM or EM, we were able to conduct a more rigorous phylogenetic inference using a partitioned analysis. Therefore, we inferred two ML phylogenies: one with two partitions representing TM and EM columns, respectively, and one with a single partition for the entire MSA. The former scheme allowed each partition to have unique distributions of evolutionary rate heterogeneity and stationary amino-acid frequencies, thus accounting for the distinct selective regimes in each domain. To ensure as much as possible that the EM and TM partitions contained only residues belonging to their respective structural domain, we created a masked MSA, in which protein residues which did not conform to their respective consensus domains were replaced with a "?". All phylogenetic analyses were conducted using this masked MSA.

We performed $100 \mathrm{ML}$ tree inferences for the unpartitioned and partitioned case each, and we compared the best resulting phylogeny from each scheme using the likelihood ratio test. We found that the partitioned model offered dramatic improvements in phylogenetic fit $\left(p<1^{-100}\right)$, highlighting the benefits of analyzing GPCRs in a structurally-aware context. We thus proceeded to analyze the partitioned phylogeny more in depth.

\section{Structurally-aware phylogeny reveals unknown biogenic amine receptor relationships and clades}

Our resulting phylogeny, shown in Figure 5, represents the most comprehensive vertebrate biogenic amine receptor phylogeny to date. This tree broadly captures many known features of biogenic amine receptor evolution, in particular that these receptors do not cluster based on ligand-binding but rather have undergone extensive functional convergent evolution. Indeed, our phylogeny indicates that only two ligand-based receptor classes, mAChR and TAAR, are truly monophyletic.

Our phylogeny features remarkably high bootstrap support for each distinct clade of receptor subtypes. We additionally find very strong support for three deeper nodes in the phylogeny that reveal the relationships among distinct receptor subtypes. The first contains the three clades HRH1, mAChR, and HRH-3,4, the second contains the clades 5HTR-1, 5HTR-5, and 5HTR-7, and the third contains the 5HTR-4 and TAAR clades. Previous studies have yielded conflicting phylogenetic placements for the 5HTR-7 clade; some have argued that 5HTR-7 is phylogenetically distinct from all other 5HTR sequences (Kakarala and Jamil 2014), while others have found evidence for a single clade containing 5HTR-5,7 as a sister taxa to a clade containing ADRA1 sequences (Fredriksson et al., 2003). Alternatively, we find moderate-to-strong support for the 5HTR-7 clade having originated before subsequent diversification into 5HTR-5 and 5HTR-1, and we find full support showing that ADRA1 forms an entirely distinct monophyletic group outside all other vertebrate biogenic amine receptors. We additionally found that $\mathrm{HRH}-3,4$ is actually a single monophyletic group. While the HRH-4 clade contains only mammalian sequences, including monotreme (platypus) sequences, HRH-3 sequences are broadly distributed across vertebrate taxa. This taxonomic distribution suggests that HRH-4 is a mammalian-specific histamine receptor class that arose from an HRH-3 duplication concurrent with mammalian origins.

In addition, among the 3039 sequences in the structurally-informed MSA, we identified 31 sequences ( $\sim 1 \%$ of our dataset) that we considered misannotated (Table 2), either because the NCBI annotation did not match the sequences' phylogenetic placement or the sequences did not cluster with known biogenic amine receptor types. Several NCBI annotations identified the correct receptor class but the incorrect receptor subtype, whereas other sequences were entirely uncharacterized. In particular, we identified an entirely unknown clade of biogenic amine receptors. This unknown clade, which appears as sister to HRH2 in Figure 5 . only contains avian sequences and a single Xenopus tropicalis (western-clawed frog) sequence. Thus, it is likely that this clade emerged concurrently with tetrapods and was secondarily lost in reptiles/birds and mammals. Interestingly, all but one of this clade's sequences were annotated in NCBI as either octopamine or No9-like receptors, both of which are insect-specific biogenic amine receptors that do not occur in vertebrate taxa (Roeder, 2005). The last sequence, alternatively, was annotated as 5HTR-7-like. Taken together, these sequence misannotations suggest an intriguing hypothesis that this clade evolved from an ancestral 5HTR sequence, and subsequent convergent evolution to insect-specific biogenic amine receptors has allowed these receptors to interact with atypical ligands for vertebrates.

\section{Dynamic lineage-specific evolution of the trace-amine associated receptors}

Of particular interest in our phylogeny are the unique evolutionary patterns revealed within the TAAR clade. While all TAAR sequences do cluster together, the TAAR phylogeny reveals the extensive expansion and contraction events characterizing this receptor family's evolution (Lindemann et al. 2005, Hashiguchi and Nishida, 2007, Stäubert et al. 2010. 2013). In fact, the TAAR subtree, displayed in Figure 6, differs somewhat from previously proposed TAAR phylogenies (Lindemann et al., 2005; Hashiguchi and Nishida, 2007) and reveals the presence of many lineage-specific subclades. 
Interestingly, only a single clade in the TAAR phylogeny, comprised of the two subclades TAAR-4 and TAAR-12, contains representatives from the full species distribution considered in our study. Further, the close phylogenetic relationship between these two subclades suggests that TAAR-4 and TAAR-12 are in fact orthologous TAAR subtypes, and the distinct naming for these subtypes apparently emerged simply because TAAR-12 is ray-finned specific. By contrast to this group, all other clades contain distinct, limited species distributions, indicative of repeated gain and loss events. In particular, the broad clade containing subtypes TAAR- $6,-7,-8$, and -9 appears to have undergone substantial lineage-specific evolution, with certain subclades only present in marsupial and placental mammals and others only present in lizards and turtles. Moreover, we identified two small clades within the broad TAAR-6,-7,-8,-9 clade that apparent indicate lineage-specific expansions specifically within bovids (labeled "B" in Figure 6) and rodents (labeled "R" in Figure 6), respectively.

Throughout the TAAR phylogeny, ray-finned and lobe-finned fish sequences frequently appear as outgroups to tetrapodspecific clades, indicating progressive diversification tracking large-scale speciation events. However, we do note that several lobe-finned fish (coelacanth) sequences are scattered across the TAAR tree and do not clearly cluster with any TAAR subtypes, likely reflecting this lineage's ancient divergence and unique evolutionary trajectory (Amemiya et al., 2013). Moreover, amphibian sequences are notably absent from this phylogeny, relative to other taxonomic groups. While absence of such sequences in our data set does not necessarily imply that these genes have actually been lost in amphibians, such a hypothesis would be consistent with the overarching gain and loss patterns that TAAR sequences display and thus may merit further study.

We additionally identified a small clade sister to TAAR (labeled in Figures 5 and 6 as TAAR ${ }^{*}$ ) that only contains sequences annotated by NCBI as "5HTR-4-like." At first glance, these annotations might suggest that 5HTR-4 is in fact paraphyletic, diversifying gradually before giving rise to TAARs. However, as all sequences in TAAR* belong taxonomically either to teleost or Xenopus tropicalis, we suspect that this clade actually corresponds to the so-called TAAR-V cluster identified by Hashiguchi and Nishida (2007). Indeed, the TAAR-V cluster contains a similar taxonomic distribution to our TAAR* and constitutes an outgroup to all other vertebrate TAAR sequences, as our phylogeny similarly displays.

Phylogenetic methods alone do not suffice to infer the evolutionary history of biogenic amine receptors Although we were able to identify several new features of biogenic amine receptor evolution, the majority of deeper splits in the phylogeny had very low bootstrap support, meaning that most of the broader relationships among biogenic amine receptors remain unresolved. This result highlights that a strictly phylogenetic approach cannot fully elucidate the complex evolutionary histories of expanding gene families. In particular, modern phylogenetic methods focus solely on the substitution process and treat MSA gaps simply as missing data. However, gaps actually represent insertion and deletion evolutionary events, and some have suggested that ignoring this information ultimately hinders phylogenetic accuracy (Morrison, 2008; Loytynoja and Goldman, 2008, Warnow, 2012; Luan et al., 2013).

This limitation is especially problematic for GPCRs. Following duplication events, GPCRs experience major indel events in their ICL and/or ECL domains, leading to dramatic shifts in loop domain sizes during the sub/neofunctionalization process. Unfortunately, the evolutionary intermediates that existed during these domain transitions have long-since disappeared from genomes, and there is no obvious way to infer the sequences of these missing links. Although the substitution process is key for understanding GPCR evolution, fully classifying relationships among GPCR families requires some understanding of how these radical domain changes occur. Therefore, additional approaches, such as syntenic analyses (Sundstrom et al., 2010; Widmark et al. 2011; Yegorov and Good, 2012, Hwang et al., 2013), combined with the phylogeny presented here should prove useful towards resolving the complete evolutionary history of vertebrate biogenic amine receptors.

\section{CONCLUSIONS}

We have established a comprehensive, structurally-informed MSA of vertebrate biogenic amine receptors. We hope that this MSA, along with its ML phylogeny, will serve as a robust resource for future studies investigating these receptors evolutionary dynamics, structural/functional constraints operating within distinct receptor clades, or overarching patterns that govern biogenic amine receptor evolution. Future work may seek to combine the analyses we have performed here with syntenic or molecular clock analyses to elucidate receptors' origin and precise evolutionary trajectories. Moreover, our MSA should prove useful in increasing accuracy in homology modeling and/or pharmaceutical development for these clinically important receptors (Kristiansen, 2004; Ishiguro, 2004; Evers et al., 2005; Mason et al., 2012).

\section{ACKNOWLEDGMENTS}

All computational resources were provided by the University of Texas at Austin's Center for Computational Biology and Bioinformatics (CCBB). We would like to thank Ahmad R. Sedaghat, MD, PhD for suggesting biogenic amine receptor evolution as a worthwhile study system. 


\section{REFERENCES}

Altschul, S., Madden, T., Schäffer, A., Zhang, J., Zhang, Z., Miller, W., and Lipman, D. (1997). Gapped BLAST and PSI-BLAST: a new generation of protein database search programs. Nucleic Acids Res, 25:3389 - 3402.

Amemiya, C. T., Alfoldi, J., Lee, A. P., Fan, S., Philippe, H., MacCallum, I., Braasch, I., Manousaki, T., Schneider, I., Rohner, N., Organ, C., Chalopin, D., Smith, J. J., Robinson, M., Dorrington, R. A., Gerdol, M., Aken, B., Biscotti, M. A., Barucca, M., Baurain, D., Berlin, A. M., Blatch, G. L., Buonocore, F., Burmester, T., Campbell, M. S., Canapa, A., Cannon, J. P., Christoffels, A., De Moro, G., Edkins, A. L., Fan, L., Fausto, A. M., Feiner, N., Forconi, M., Gamieldien, J., Gnerre, S., Gnirke, A., Goldstone, J. V., Haerty, W., Hahn, M. E., Hesse, U., Hoffmann, S., Johnson, J., Karchner, S. I., Kuraku, S., Lara, M., Levin, J. Z., Litman, G. W., Mauceli, E., Miyake, T., Mueller, M. G., Nelson, D. R., Nitsche, A., Olmo, E., Ota, T., Pallavicini, A., Panji, S., Picone, B., Ponting, C. P., Prohaska, S. J., Przybylski, D., Saha, N. R., Ravi, V., Ribeiro, F. J., Sauka-Spengler, T., Scapigliati, G., Searle, S. M. J., Sharpe, T., Simakov, O., Stadler, P. F., Stegeman, J. J., Sumiyama, K., Tabbaa, D., Tafer, H., Turner-Maier, J., van Heusden, P., White, S., Williams, L., Yandell, M., Brinkmann, H., Volff, J.-N., Tabin, C. J., Shubin, N., Schartl, M., Jaffe, D. B., Postlethwait, J. H., Venkatesh, B., Di Palma, F., Lander, E. S., Meyer, A., and Lindblad-Toh, K. (2013). The african coelacanth genome provides insights into tetrapod evolution. Nature, 496:311 - 316.

Anbazhagan, P., Purushottam, M., Kiran Kumar, H. B., Mukherjee, O., Jain, S., and Sowdhamini, R. (2010). Phylogenetic analysis and selection pressures of 5-HT receptors in human and non-human primates: Receptor of an ancient neurotransmitter. J Biomol Struct Dyn, 27(5):581 - 598.

Bockaert, J. and Pin, J. P. (1999). Molecular tinkering of G protein-coupled receptors: an evolutionary success. EMBO J, $18(7): 1723-1729$.

Callier, S., Snapyan, M., Crom, S., Prou, D., Vincent, J.-D., and Vernier, P. (2003). Evolution and cell biology of dopamine receptors in vertebrates. Biol Cell, 95(7):489- 502.

Chang, J.-M., Di Tommaso, P., Taly, J.-F., and Notredame, C. (2012). Accurate multiple sequence alignment of transmembrane proteins with PSI-Coffee. BMC Bioinformatics, 13(Suppl 4):S1.

Eddy, S. (1998). Profile hidden Markov models. Bioinformatics, 14:755 - 763.

Evers, A., Hessler, G., Matter, H., and Klabunde, T. (2005). Virtual screening of biogenic amine-binding G-protein coupled receptors: Comparative evaluation of protein- and ligand-based virtual screening protocols. J Med Chem, 48(17):5448 5465.

Franzosa, E., Xue, R., and Xia, Y. (2013). Quantitative residue-level structure-evolution relationships in the yeast membrane proteome. Genome Biol Evol, 5:734 - 744.

Fredriksson, R., Lagerstrom, M., Lundin, L., and Schioth, H. (2003). The G-protein-coupled receptors in the human genome form five main families. phylogenetic analysis, paralogon groups, and fingerprints. Mol Pharmacol, 63(6):1256 - 1272.

Gloriam, D. E. I., Bjarnadóttir, T. K., Yan, Y.-L., Postlethwait, J. H., Schioth, H. B., and Fredriksson, R. (2005). The repertoire of trace amine G-protein-coupled receptors: large expansion in zebrafish. Mol Phylogenet Evol, 35(2):470 482.

Hashiguchi, Y. and Nishida, M. (2007). Evolution of trace amine associated receptor (taar) gene family in vertebrates: Lineage-specific expansions and degradations of a second class of vertebrate chemosensory receptors expressed in the olfactory epithelium. Mol Biol Evol, 24(9):2099 - 2107.

Hill, J. R. and Deane, C. M. (2012). MP-T: improving membrane protein alignment for structure prediction. Bioinformatics, 29(1):54-61.

Hwang, J. I., Moon, M. J., Park, S., Kim, D. K., Cho, E. B., Ha, N., Son, G. H., Kim, K., Vaudry, H., and Seong, J. Y. (2013). Expansion of secretin-like G protein-coupled receptors and their peptide ligands via local duplications before and after two rounds of whole-genome duplication. Mol Biol Evol, 30(5):1119 - 1130.

Ishiguro, M. (2004). Ligand-binding modes in cationic biogenic amine receptors. ChemBioChem, 5(9):1210 - 1219.

Jordan, G. and Goldman, N. (2012). The effects of alignment error and alignment filtering on the sitewise detection of positive selection. Mol Biol Evol, 29:1125 - 1139.

Julenius, K. and Pedersen, A. G. (2006). Protein evolution is faster outside the cell. Mol Biol Evol, 23:2039 - 2048.

Kakarala, K. K. and Jamil, K. (2014). Sequence-structure based phylogeny of GPCR class A rhodopsin receptors. Mol Phylogenet Evol, 74(C):66 - 96.

Katoh, K. and Standley, D. M. (2013). MAFFT multiple sequence alignment software version 7: Improvements in performance and usability. Mol Biol Evol, 30:772 - 780.

Krishnan, A., Almén, M. S., Fredriksson, R., and Schioth, H. B. (2012). The origin of GPCRs: Identification of mammalian like rhodopsin, adhesion, glutamate and frizzled GPCRs in fungi. PLoS ONE, 7(1):e29817.

Kristiansen, K. (2004). Molecular mechanisms of ligand binding, signaling, and regulation within the superfamily of Gprotein-coupled receptors: molecular modeling and mutagenesis approaches to receptor structure and function. Pharmacol 
Therapeut, 103(1):21 - 80.

Lagerstrom, M. C. and Schioth, H. B. (2008). Structural diversity of G protein-coupled receptors and significance for drug discovery. Nat Rev Drug Discov, 7:339 - 357.

Le, S. and Gascuel, O. (2008). An improved general amino acid replacement matrix. Mol Biol Evol, $25: 1307$ - 1320.

Lindemann, L., Ebeling, M., Kratochwil, N. A., Bunzow, J. R., Grandy, D. K., and Hoener, M. C. (2005). Trace amine-associated receptors form structurally and functionally distinct subfamilies of novel G protein-coupled receptors. Genomics, 85(3):372 - 385.

Loytynoja, A. and Goldman, N. (2008). Phylogeny-aware gap placement prevents errors in sequence alignment and evolutionary analysis. Science, 320:1632 - 1635.

Luan, P., Ryder, O. A., Davis, H., and Zhang, Y. (2013). Incorporating indels as phylogentic characters: impact for interfamilial relationships within Arctoidea (Mammalia: Carnivora). Mol Phylogenet Evol, 66:748 - 756.

Mason, J. S., Bortolato, A., Congreve, M., and Marshall, F. H. (2012). New insights from structural biology into the druggability of G protein-coupled receptors. Trends in Pharmacol Sci, 33(5):249- 260.

Morrison, D. A. (2008). A framework for phylogenetic sequence alignment. Plant Syst Evol, 282(3-4):127 - 149.

Nichols, D. E. and Nichols, C. D. (2008). Serotonin receptors. Chemical Reviews, 108(5):1614 - 1641.

Oberai, A., Joh, N. H., Pettit, F. K., and Bowie, J. U. (2009). Structural imperatives impose diverse evolutionary constraints on helical membrane proteins. Proc Natl Acad Sci USA, 106:17747 - 17750.

Ogden, T. H. and Rosenberg, M. S. (2006). Multiple sequence alignment accuracy and phylogenetic inference. Syst Biol, $55(2): 314-328$.

O’Sullivan, O., Suhre, K., Abergel, C., Higgins, D. G., and Notredame, C. (2004). 3DCoffee: Combining protein sequences and structures within multiple sequence alignments. J Mol Biol, 340:385 - 395.

Pei, J., Kim, B. H., and Grishin, N. V. (2008). PROMALS3D: a tool for multiple protein sequence and structure alignments. Nuc Acids Res, 36(7):2295 - 2300.

Pruitt, K. D., Brown, G. R., Hiatt, S. M., Thibaud-Nissen, F., Astashyn, A., Ermolaeva, O., Farrell, C. M., Hart, J., Landrum, M. J., McGarvey, K. M., Murphy, M. R., O’Leary, N. A., Pujar, S., Rajput, B., Rangwala, S. H., Riddick, L. D., Shkeda, A., Sun, H., Tamez, P., Tully, R. E., Wallin, C., Webb, D., Weber, J., Wu, W., DiCuccio, M., Kitts, P., Maglott, D. R., Murphy, T. D., and Ostell, J. M. (2013). RefSeq: an update on mammalian reference sequences. Nucleic Acids Res, 42(D1):D756-D763.

Roeder, T. (2005). Tyramine and octopamine: Ruling behavior and metabolism. Annual Review of Entomology, 50(1):447 477.

Schoneberg, T., Schulz, A., Biebermann, H., Hermsdorf, T., H, R., and Sangkuhl, K. (2004). Mutant G protein-coupled receptors as a cause of human diseases. Pharmacol Ther, 104:173 - 206.

Spielman, S. J. and Wilke, C. O. (2013). Membrane environment imposes unique selection pressures on transmembrane domains of G protein-coupled receptors. J Mol Evol, 76(3):172 - 182.

Stamatakis, A. (2006). Phylogenetic models of rate heterogeneity: a high performance computing perspective. In Proc. of IPDPS2006.

Stamatakis, A. (2014). RAxML Version 8: A tool for phylogenetic analysis and post-analysis of large phylogenies. Bioinformatics, 30(9):1312 - 1313.

Stäubert, C., Böselt, I., Bohnekamp, J., Rompler, H., Enard, W., and Schöneberg, T. (2010). Structural and functional evolution of the trace amine-associated receptors TAAR3, TAAR4 and TAAR5 in primates. PLoS ONE, 5(6):e11133.

Stäubert, C., Le Duc, D., and Schöneberg, T. (2013). Examining the dynamic evolution of G protein-coupled receptors. In $G$ protein-coupled receptor genetics: research and methods in the post-genomic era, pages 23 - 43 . Springer, Totowa, NJ.

Stevens, T. J. and Arkin, I. T. (2001). Substitution rates in alpha-helical transmembrane proteins. Prot Sci, 10:2507 - 2517.

Sundstrom, G., Dreborg, S., and Larhammar, D. (2010). Concomitant duplications of opioid peptide and receptor genes before the origin of jawed vertebrates. PLoS One, 5:e10512.

Tourasse, N. J. and Li, W.-H. (2000). Selective constraints, amino acid composition, and the rate of protein evolution. Mol Biol Evol, 17:656 - 664 .

Vassilatis, D., Hohmann, J., Zeng, H., Li, F., Ranchalis, J., Mortrud, M., Brown, A., Rodriguez, S., Weller, J., Wright, A., Bergmann, J., and Gaitanaris, G. (2003). The G protein-coupled receptor repertoires of human and mouse. Proc Natl Acad Sci USA, 100(8):4903 - 4908.

Warnow, T. (2012). Standard maximum likelihood analyses of alignments with gaps can be statistically inconsistent. PLoS Curr, 4:RRN1308.

Widmark, J., Sundstrom, G., Ocampo, D., and Larhammar, D. (2011). Differential evolution of voltage-gated sodium channels in tetrapods and teleost fishes. Mol Biol Evol, 28:859 - 871.

Wistrand, M., Käll, L., and Sonnhammer, E. L. L. (2006). A general model of G protein-coupled receptor sequences and its 
application to detect remote homologs. Prot Sci, 15(3):509- 521.

Wong, K. M., Suchard, M. A., and Huelsenbeck, J. P. (2008). Alignment uncertainty and genomic analysis. Science, 319(5862):473 - 476.

Yamamoto, K., Mirabeau, O., Bureau, C., Blin, M., Michon-Coudouel, S., Demarque, M., and Vernier, P. (2013). Evolution of dopamine receptor genes of the D1 class in vertebrates. Mol Biol Evol, 30(4):833 - 843.

Yegorov, S. and Good, S. (2012). Using paleogenomics to study the evolution of gene families: Origin and duplication history of the relaxin family hormones and their receptors. PLoS ONE, 7(3):e32923. 
FIGURES AND TABLES

\begin{tabular}{lll}
\hline \multicolumn{1}{c}{ Receptor Class } & Abbreviation & N \\
\hline Serotonin receptors & 5HTR & 972 \\
Adrenergic receptors & ADR & 611 \\
Dopamine receptors & DRD & 464 \\
Muscarinic cholinergic receptors & mAChR & 353 \\
Trace amine-associated receptors & TAAR & 343 \\
Histamine receptors & HRH & 286 \\
Unknown receptors & Unknown & 10 \\
\hline
\end{tabular}

Table 1. Biogenic amine receptor classes, and their abbreviations, considered in this study. The receptor class "Unknown" refers to the corresponding uncharacterized clade in Figure 5, and the column " $\mathrm{N}$ " indicates the total number of sequences for each broad receptor class in our structurally-informed MSA. 


\begin{tabular}{|c|c|c|c|}
\hline Protein ID & Nucleotide ID & Current Classification & Proposed Classification \\
\hline XP_005797918.1 & XM_005797861.1 & DRD-2 & DRD-3 \\
\hline XP_003967971.1 & XM_003967922.1 & DRD-2 & DRD-3 \\
\hline NP_001266433.1 & NM_001279504.1 & mAChR-4 & mAChR-2 \\
\hline XP_001520508.2 & XM_001520458.3 & HRH-3 & HRH-4 \\
\hline XP_005282846.1 & XM_005282789.1 & HRH-4 & HRH-3 \\
\hline XP_001920844.1 & XM_001920809.1 & TAAR-4-like & TAAR-12 \\
\hline NP_001076571.1 & NM_001083102.1 & TAAR-64 & TAAR-13 \\
\hline XP_006014096.1 & XM_006014034.1 & TAAR-9-like & TAAR-4 \\
\hline XP_003201718.2 & XM_003201670.2 & TAAR-1-like & TAAR-10 \\
\hline NP_001076546.1 & NM_001083077.1 & TAAR-11-like & TAAR-10 \\
\hline NP_001083418.1 & NM_001089949.1 & uncharacterized & ADRB \\
\hline NP_001103208.1 & NM_001109738.1 & uncharacterized & HRH-2 \\
\hline NP_001124143.1 & NM_001130671.1 & uncharacterized & TAAR-12 \\
\hline XP_001337671.1 & XM_001337635.2 & 5HTR-4-like & TAAR $^{*}$ \\
\hline XP_003976403.1 & XM_003976354.1 & 5HTR-4-like & TAAR* \\
\hline XP_005810466.1 & XM_005810409.1 & 5HTR-4-like & TAAR $^{*}$ \\
\hline XP_003454279.1 & XM_003454231.1 & 5HTR-4-like & TAAR* \\
\hline XP_004549625.1 & XM_004549568.1 & 5HTR-4-like & TAAR* \\
\hline XP_002935532.2 & XM_002935486.2 & 5HTR-4-like & TAAR* \\
\hline XP_006013317.1 & XM_006013255.1 & 5HTR-4-like & TAAR* \\
\hline XP_005510029.1 & XM_005509972.1 & 5HTR-7-like & Unknown \\
\hline XP_002187301.2 & XM_002187265.2 & Octopamine receptor-like & Unknown \\
\hline XP_002937327.2 & XM_002937281.2 & Octopamine receptor-like & Unknown \\
\hline XP_005045681.1 & XM_005045624.1 & Octopamine receptor-like & Unknown \\
\hline XP_005144673.1 & XM_005144616.1 & Octopamine receptor-like & Unknown \\
\hline XP_005229932.1 & XM_005229875.1 & Octopamine receptor-like & Unknown \\
\hline XP_005428400.1 & XM_005428343.1 & Probable GPCR No9-like & Unknown \\
\hline XP_005490920.1 & XM_005490863.1 & Probable GPCR No9-like & Unknown \\
\hline XP_005518128.1 & XM_005518071.1 & Probable GPCR No9-like & Unknown \\
\hline XP_006111669.1 & XM_006111607.1 & Octopamine receptor-like & Unknown \\
\hline XP_420867.2 & XM_420867.4 & Octopamine receptor & Unknown \\
\hline
\end{tabular}

Table 2. Misannotated and uncharacterized sequences identified through phylogenetic analysis. Based on sequence placement in the structurally-partitioned phylogeny, we propose updated classifications for 31 biogenic amine receptor sequences. The proposed classifications "Unknown" and "TAAR" refer to the correspondingly-labeled clades in Figure 5 

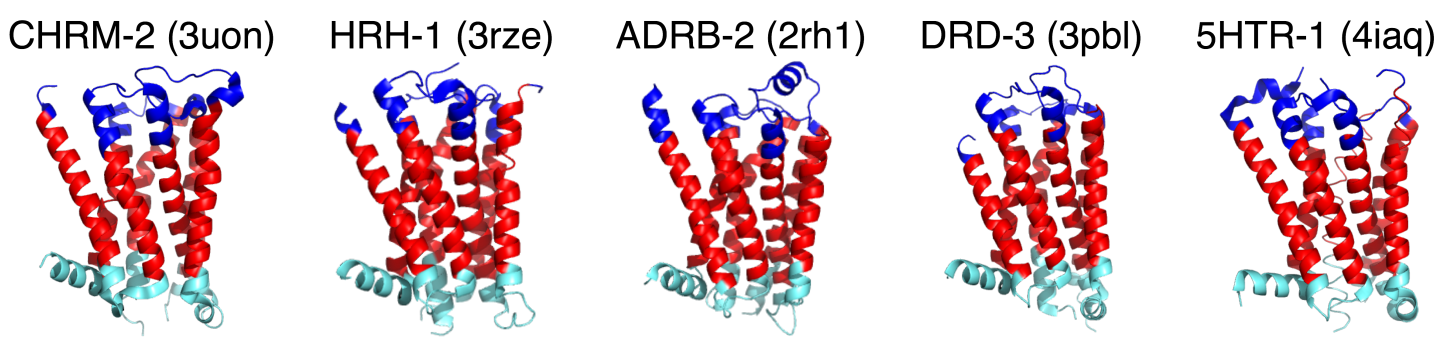

Figure 1. GPCRHMM domain predictions for representative biogenic amine receptor crystal structures from the Protein Data Bank (PDB). Gene names are shown in capital letters above each structure, and corresponding PDB IDs are shown in parentheses. Dark blue represents predicted extracellular residues, red represents predicted TM residues, light blue represents predicted intracellular residues.
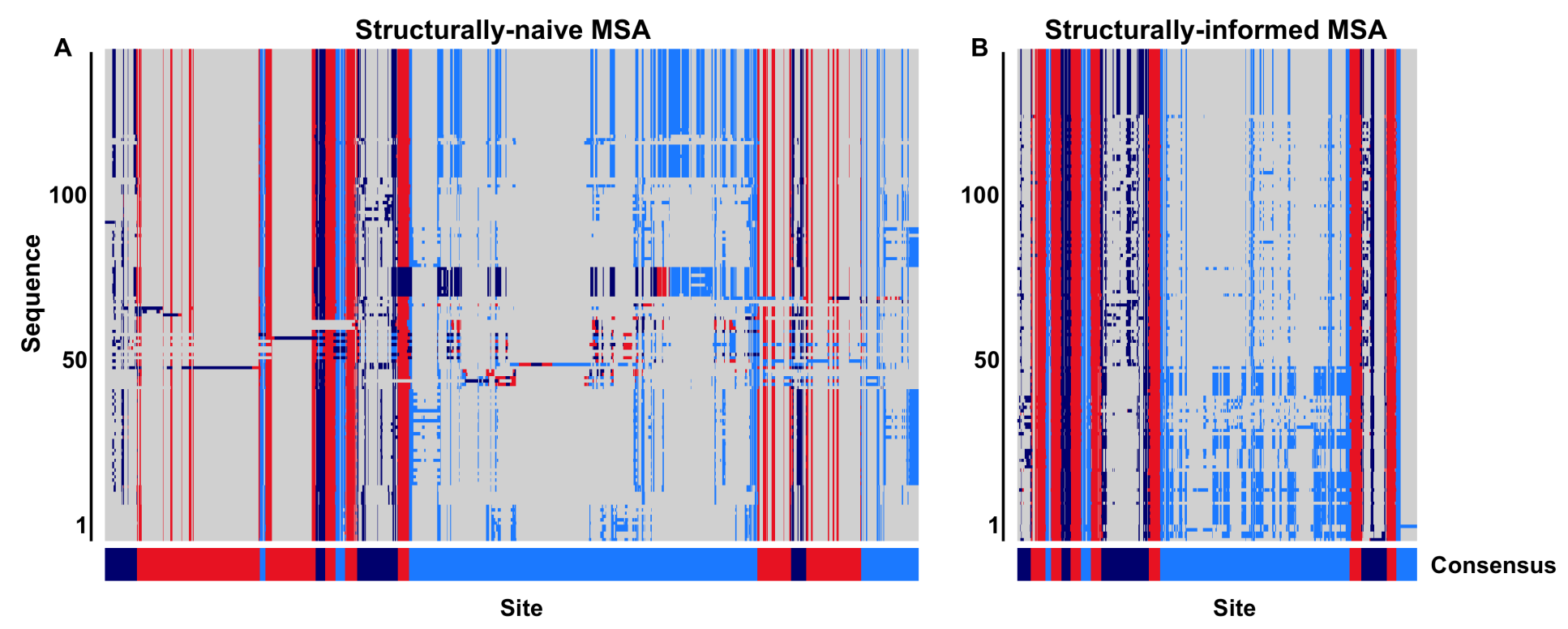

Figure 2. Graphical representation of a subset of the A) structurally-naive and B) structurally-informed biogenic amine receptor MSAs. Each panel displays 130 MSA rows focused specifically on the MSA section containing the seven TM domains. Dark blue represents predicted extracellular residues, red represents predicted TM residues, lighter blue represents predicted intracellular residues, and gray represents MSA gaps. The bottom bar below each MSA figure shows the consensus domain structure for each MSA. The structurally-naive MSA was built all 3464 putative GPCR sequences in MAFFT, whereas the structurally-informed MSA was built using the iterative strategy outlined in Figure 3 Note that all columns which contain only gaps in this MSA subset have been removed from this figure for visual clarity. 


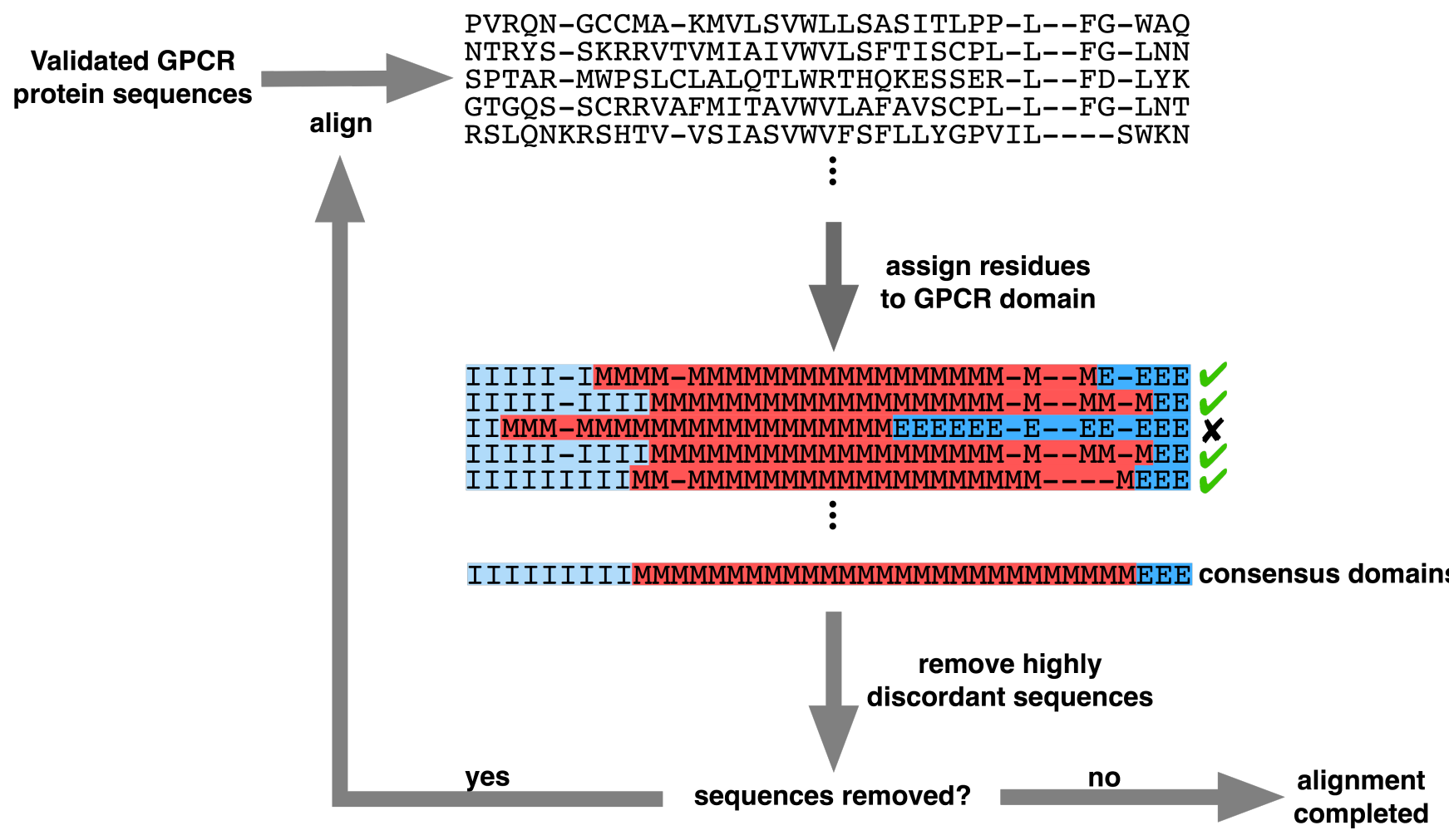

Figure 3. Iterative alignment strategy used to generate the structurally-informed vertebrate biogenic amine receptor MSA. A total of 3464 sequences were initially input ("Validated GPCR protein sequences"), and the final structurally-informed MSA contained 3039 protein sequences. Residues marked with "I" represent intracellular residues, those marked with "M" represent TM residues, and those marked with "E" represent extracellular residues. MSA gaps were treated as missing data when determining each column's consensus structural domain. Sequenced were removed ("remove highly discordant sequences") if $\geq 5 \%$ of columns belonged to a different structural domain than the respective consensus domain. Note that the MSA shown in this figure represents a subset of the entire MSA. 


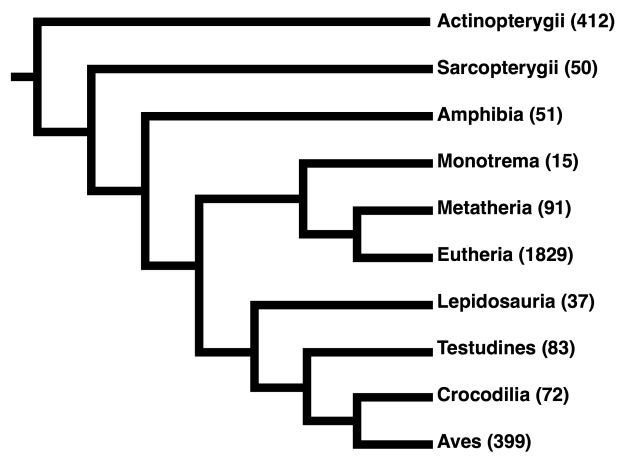

Figure 4. Cladogram of the taxonomic distribution of all sequences in the final structurally-informed MSA. All sequences belonged to the Euteleostomi clade of jawed vertebrates. Numbers in parentheses indicate the total number of sequences from the respective clade. We note that our MSA is particularly enriched for sequences from Eutherian (placental mammal) species, likely due to the stringent filters we applied to sequence collection that favored fully-sequenced genomes. 


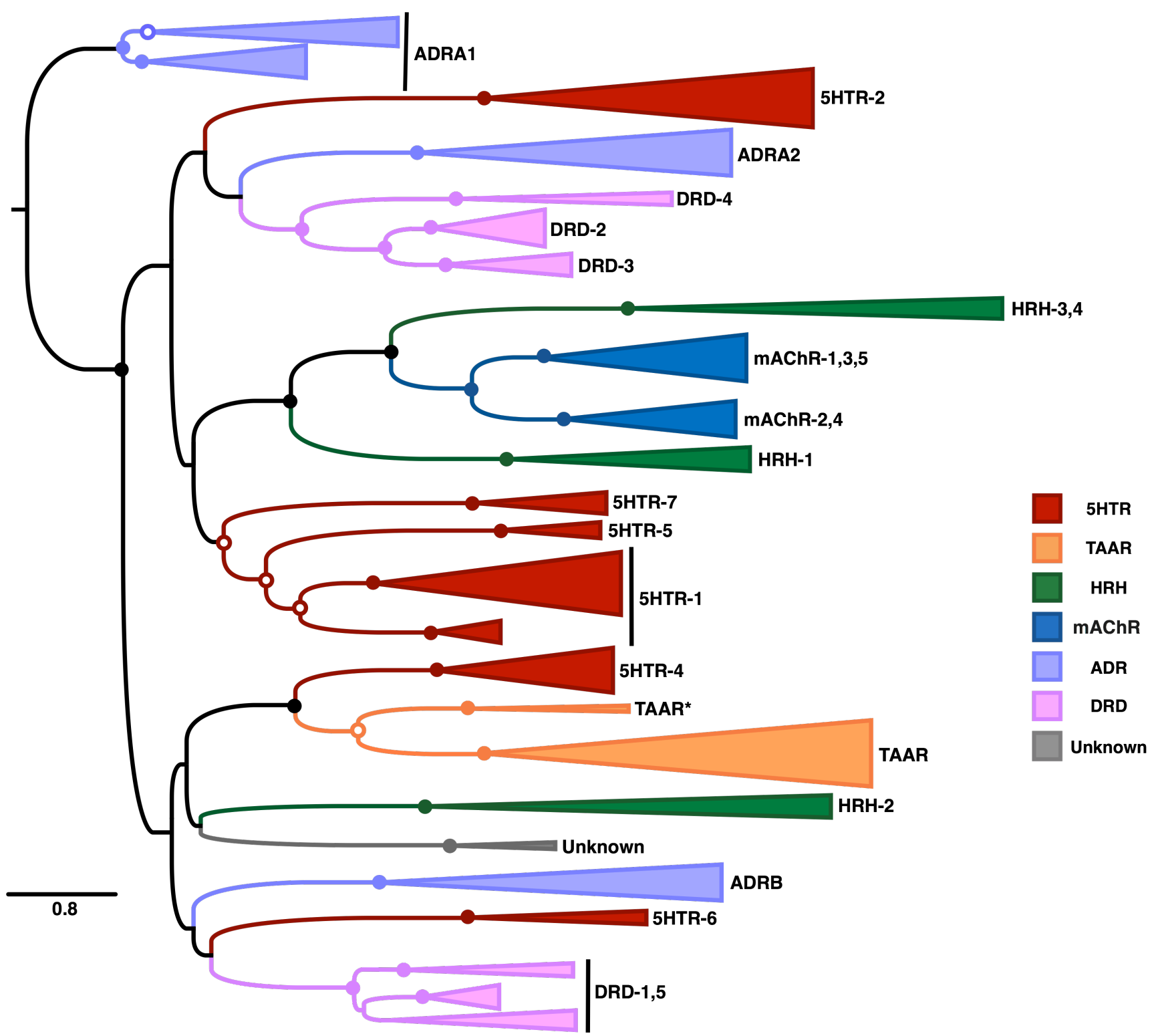

Figure 5. Maximum-likelihood phylogeny of vertebrate biogenic amine receptors built using the masked structurally-informed MSA in RAxML. Nodes with open circles indicate $\geq 50 \%$ bootstrap support, and nodes with closed circles indicate $\geq 90 \%$ bootstrap support. Biogenic amine receptors are abbreviated as in Table 1. The clade labeled "Unknown" could not be clearly identified as one of the major receptor types and may represent a previously unrecognized biogenic amine receptor clade. Note that the root shown on this tree has been placed arbitrarily. 


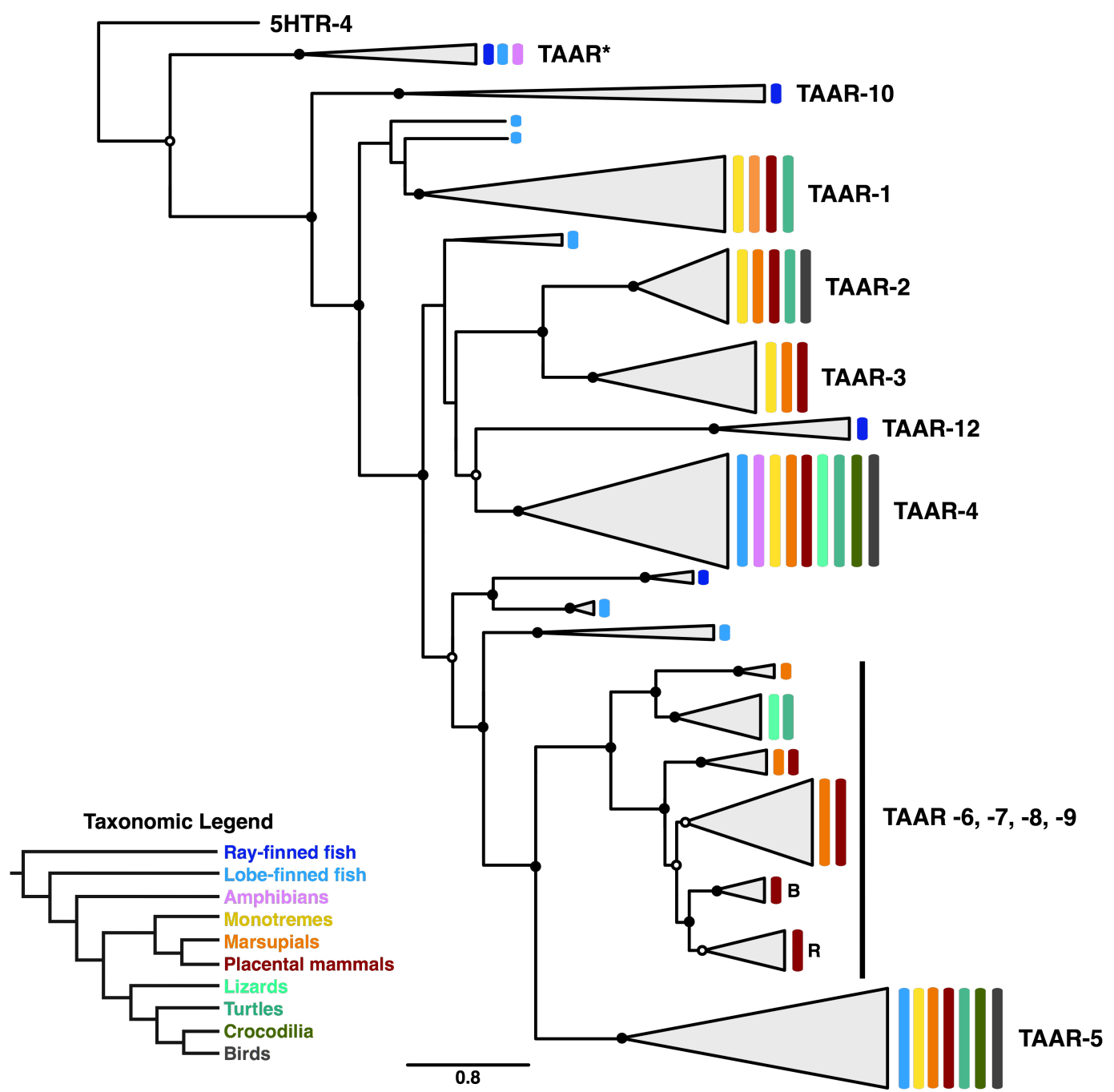

Figure 6. Subclade of the TAAR receptors within the phylogeny shown in Figure 5 . Nodes with open circles indicate $\geq 50 \%$ bootstrap support, and nodes with closed circles indicate $\geq 90 \%$ bootstrap support. The subclades within the TAAR-6,7,8,9 clade labeled as "B" and "R" indicate clades containing only bovid and rodent sequences, respectively. 
Table $\mathbf{1}$ (on next page)

Biogenic amine receptor classes, and their abbreviations, considered in this study.

The receptor class "Unknown" refers to the corresponding uncharacterized clade in Figure 5, and the column " $\mathrm{N}$ " indicates the total number of sequences for each broad receptor class in our structurally-informed MSA. 


\begin{tabular}{|l|l|l|}
\hline \multicolumn{1}{|c|}{ Receptor Class } & Abbreviation & \multicolumn{1}{c|}{ N } \\
\hline Serotonin receptors & $5 \mathrm{HTR}$ & $\begin{array}{l}97 \\
2\end{array}$ \\
\hline Adrenergic receptors & ADR & $\begin{array}{l}61 \\
1\end{array}$ \\
\hline Dopamine receptors & DRD & $\begin{array}{l}46 \\
4\end{array}$ \\
\hline $\begin{array}{l}\text { Muscarinic cholinergic } \\
\text { receptors }\end{array}$ & mAChR & $\begin{array}{l}35 \\
3\end{array}$ \\
\hline $\begin{array}{l}\text { Trace amine-associated } \\
\text { receptors }\end{array}$ & TAAR & 34 \\
\hline Histamine receptors & HRH & 28 \\
& & 6 \\
\hline Unknown receptors & Unknown & 10 \\
\hline
\end{tabular}


Table 2 (on next page)

Misannotated and uncharacterized sequences identified through phylogenetic analysis.

Based on sequence placement in the structurally-partitioned phylogeny, we propose updated classifications for 31 biogenic amine receptor sequences. The proposed classifications "Unknown" and "TAAR*" refer to the correspondingly-labeled clades in Figure 5. 


\begin{tabular}{|c|c|c|c|}
\hline Protein ID & Nucleotide ID & $\begin{array}{l}\text { Current } \\
\text { Classification }\end{array}$ & $\begin{array}{l}\text { Proposed } \\
\text { Classification }\end{array}$ \\
\hline XP_005797918.1 & XM_005797861.1 & DRD-2 & DRD-3 \\
\hline XP_003967971.1 & XM_003967922.1 & DRD-2 & DRD-3 \\
\hline NP 001266433.1 & NM 001279504.1 & mAChR-4 & mAChR-2 \\
\hline XP 001520508.2 & XM 001520458.3 & HRH-3 & $\mathrm{HRH}-4$ \\
\hline XP_005282846.1 & XM_005282789.1 & $\mathrm{HRH}-4$ & $\mathrm{HRH}-3$ \\
\hline XP_001920844.1 & XM_001920809.1 & TAAR-4-like & TAAR-12 \\
\hline NP_001076571.1 & NM_001083102.1 & TAAR-64 & TAAR-13 \\
\hline XP_006014096.1 & XM_006014034.1 & TAAR-9-like & TAAR-4 \\
\hline XP_003201718.2 & XM_003201670.2 & TAAR-1-like & TAAR-10 \\
\hline NP_001076546.1 & NM_001083077.1 & TAAR-11-like & TAAR-10 \\
\hline NP_001083418.1 & NM_001089949.1 & uncharacterized & ADRB \\
\hline NP_001103208.1 & NM_001109738.1 & uncharacterized & $\mathrm{HRH}-2$ \\
\hline NP_001124143.1 & NM_001130671.1 & uncharacterized & TAAR-12 \\
\hline XP_001337671.1 & XM_001337635.2 & 5HTR-4-like & TAAR $^{*}$ \\
\hline XP_003976403.1 & XM_003976354.1 & 5HTR-4-like & TAAR $^{*}$ \\
\hline XP_005810466.1 & XM_005810409.1 & 5HTR-4-like & TAAR $^{*}$ \\
\hline XP_003454279.1 & XM_003454231.1 & 5HTR-4-like & TAAR $^{*}$ \\
\hline XP_004549625.1 & XM_004549568.1 & 5HTR-4-like & TAAR $^{*}$ \\
\hline XP_002935532.2 & XM_002935486.2 & 5HTR-4-like & TAAR $^{*}$ \\
\hline XP_006013317.1 & XM_006013255.1 & 5HTR-4-like & TAAR $^{*}$ \\
\hline XP_005510029.1 & XM_005509972.1 & 5HTR-7-like & Unknown \\
\hline XP_002187301.2 & XM_002187265.2 & $\begin{array}{l}\text { Octopamine receptor- } \\
\text { like }\end{array}$ & Unknown \\
\hline XP_002937327.2 & XM_002937281.2 & $\begin{array}{l}\text { Octopamine receptor- } \\
\text { like }\end{array}$ & Unknown \\
\hline XP_005045681.1 & XM_005045624.1 & $\begin{array}{l}\text { Octopamine receptor- } \\
\text { like }\end{array}$ & Unknown \\
\hline XP_005144673.1 & XM_005144616.1 & $\begin{array}{l}\text { Octopamine receptor- } \\
\text { like }\end{array}$ & Unknown \\
\hline XP_005229932.1 & XM_005229875.1 & $\begin{array}{l}\text { Octopamine receptor- } \\
\text { like }\end{array}$ & Unknown \\
\hline XP_005428400.1 & XM_005428343.1 & $\begin{array}{l}\text { Probable GPCR No9- } \\
\text { like }\end{array}$ & Unknown \\
\hline XP_005490920.1 & XM_005490863.1 & $\begin{array}{l}\text { Probable GPCR No9- } \\
\text { like }\end{array}$ & Unknown \\
\hline XP_005518128.1 & XM_005518071.1 & $\begin{array}{l}\text { Probable GPCR No9- } \\
\text { like }\end{array}$ & Unknown \\
\hline XP_006111669.1 & XM_006111607.1 & $\begin{array}{l}\text { Octopamine receptor- } \\
\text { like }\end{array}$ & Unknown \\
\hline XP 420867.2 & XM_420867.4 & Octopamine receptor & Unknown \\
\hline
\end{tabular}




\section{1}

GPCRHMM domain predictions for representative biogenic amine receptor crystal structures from the Protein Data Bank (PDB).

Gene names are shown in capital letters above each structure, and corresponding PDB IDs are shown in parentheses. Dark blue represents predicted extracellular residues, red represents predicted TM residues, light blue represents predicted intracellular residues.

CHRM-2 (3uon)

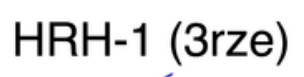

ADRB-2 (2rh1)

DRD-3 (3pbl)

5HTR-1 (4iaq)
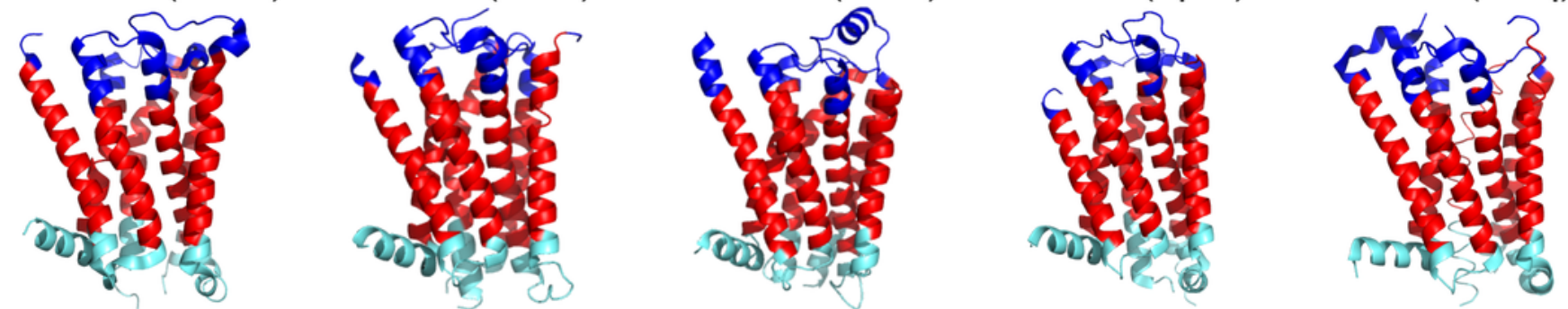
Graphical representation of a subset of the A) structurally-naive and B) structurallyinformed biogenic amine receptor MSAs.

Each panel displays 130 MSA rows focused specifically on the MSA section containing the seven TM domains. Dark blue represents predicted extracellular residues, red represents predicted TM residues, lighter blue represents predicted intracellular residues, and gray represents MSA gaps. The bottom bar below each MSA figure shows the consensus domain structure for each MSA. The structurally-naive MSA was built all 3464 putative GPCR sequences in MAFFT, whereas the structurally-informed MSA was built using the iterative strategy outlined in Figure 3. Note that all columns which contain only gaps in this MSA subset have been removed from this figure for visual clarity.
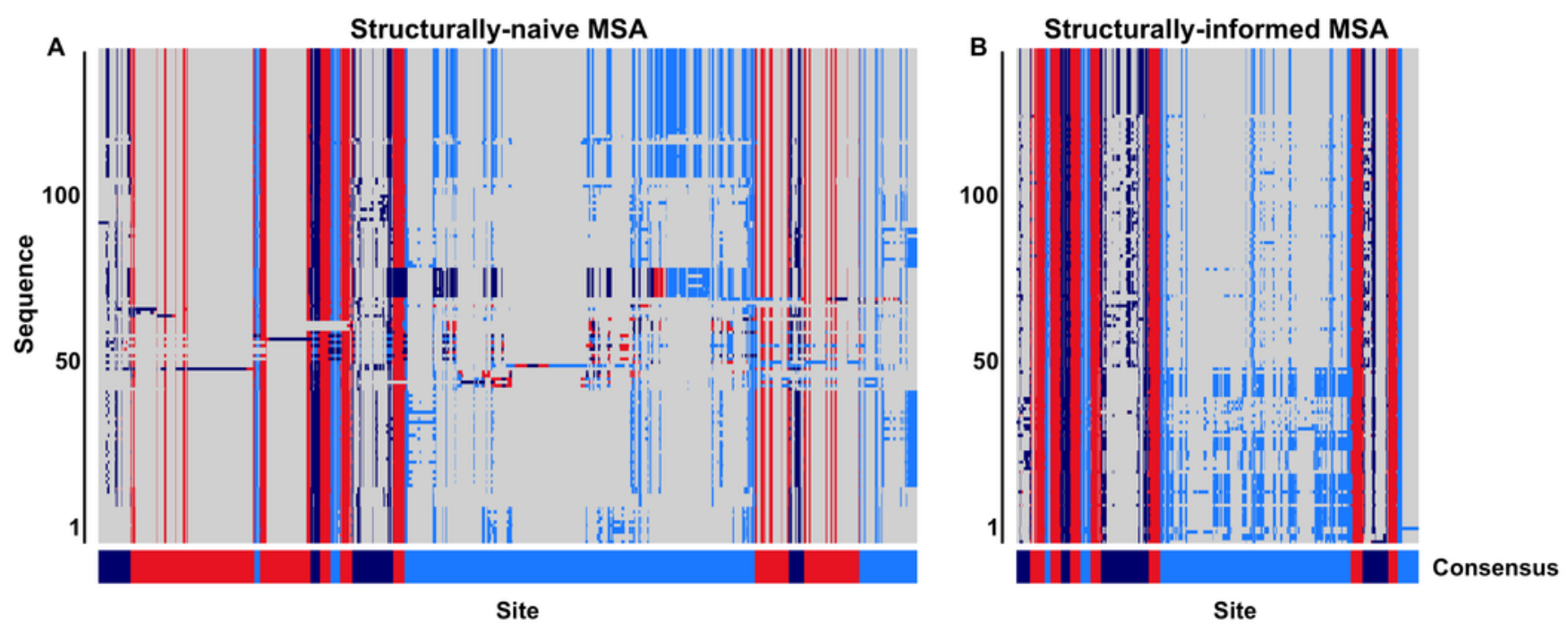
3

Iterative alignment strategy used to generate the structurally-informed vertebrate biogenic amine receptor MSA.

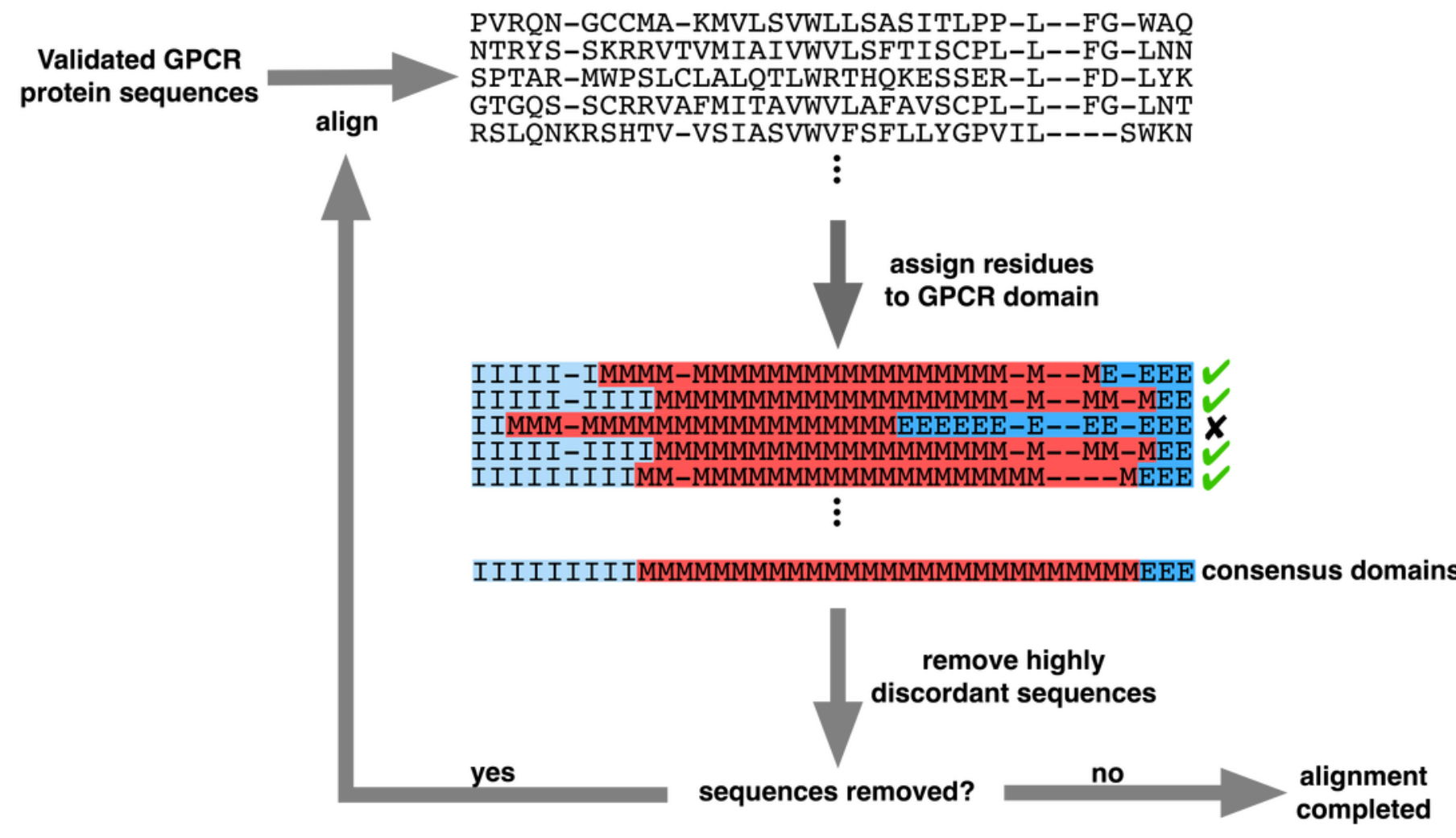


4

Cladogram of the taxonomic distribution of all sequences in the final structurallyinformed MSA.

All sequences belonged to the Euteleostomi clade of jawed vertebrates. Numbers in parentheses indicate the total number of sequences from the respective clade. We note that our MSA is particularly enriched for sequences from Eutherian (placental mammal) species, likely due to the stringent filters we applied to sequence collection that favored fullysequenced genomes.

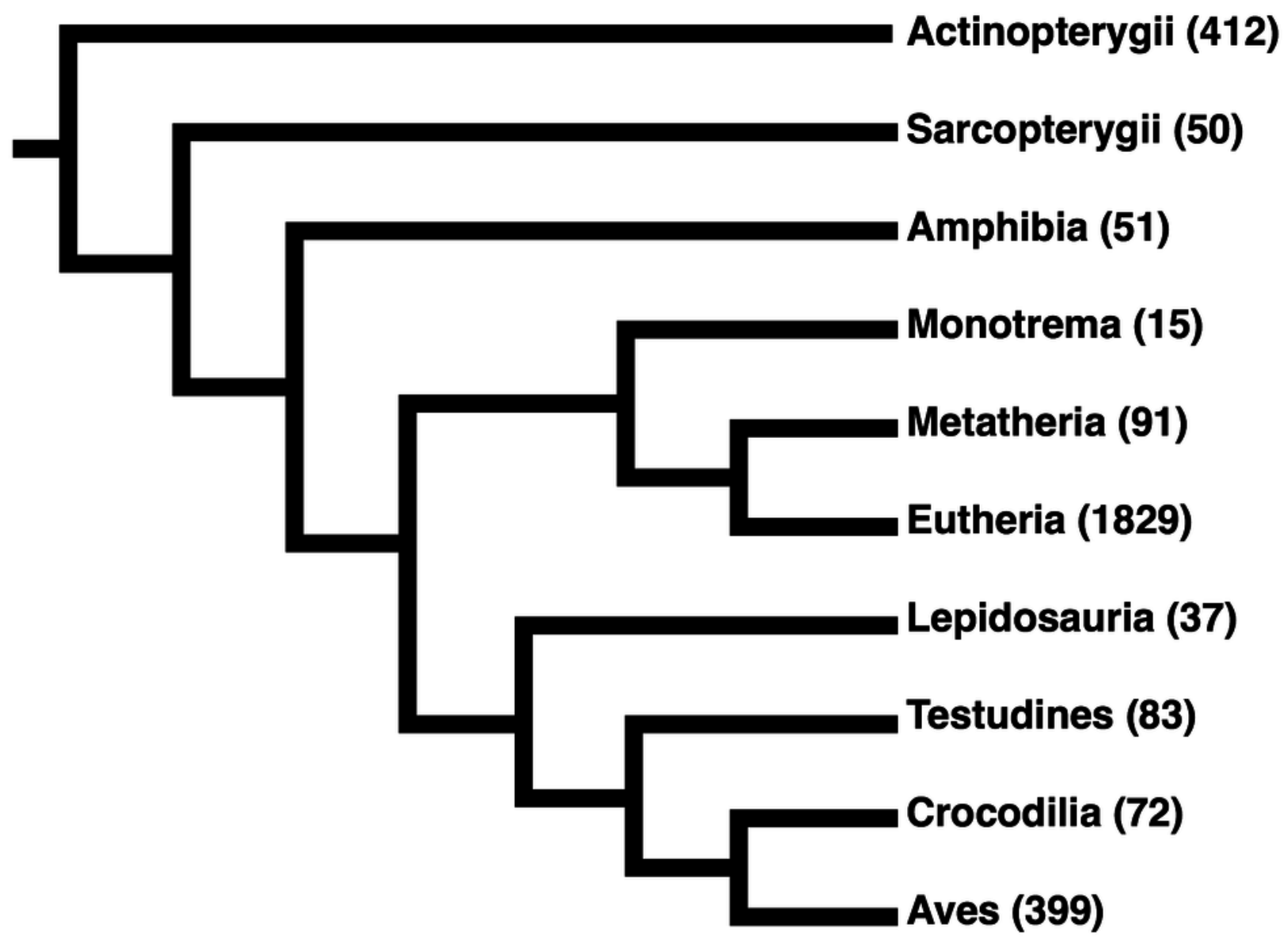




\section{5}

Maximum-likelihood phylogeny of vertebrate biogenic amine receptors built using the masked structurally-informed MSA in RAxML.

Nodes with open circles indicate $\geq 50 \%$ bootstrap support, and nodes with closed circles indicate $\geq 90 \%$ bootstrap support. Biogenic amine receptors are abbreviated as in Table 1 . The clade labeled "Unknown" could not be clearly identified as one of the major receptor types and may represent a previously unrecognized biogenic amine receptor clade. Note that the root shown on this tree has been placed arbitrarily. 


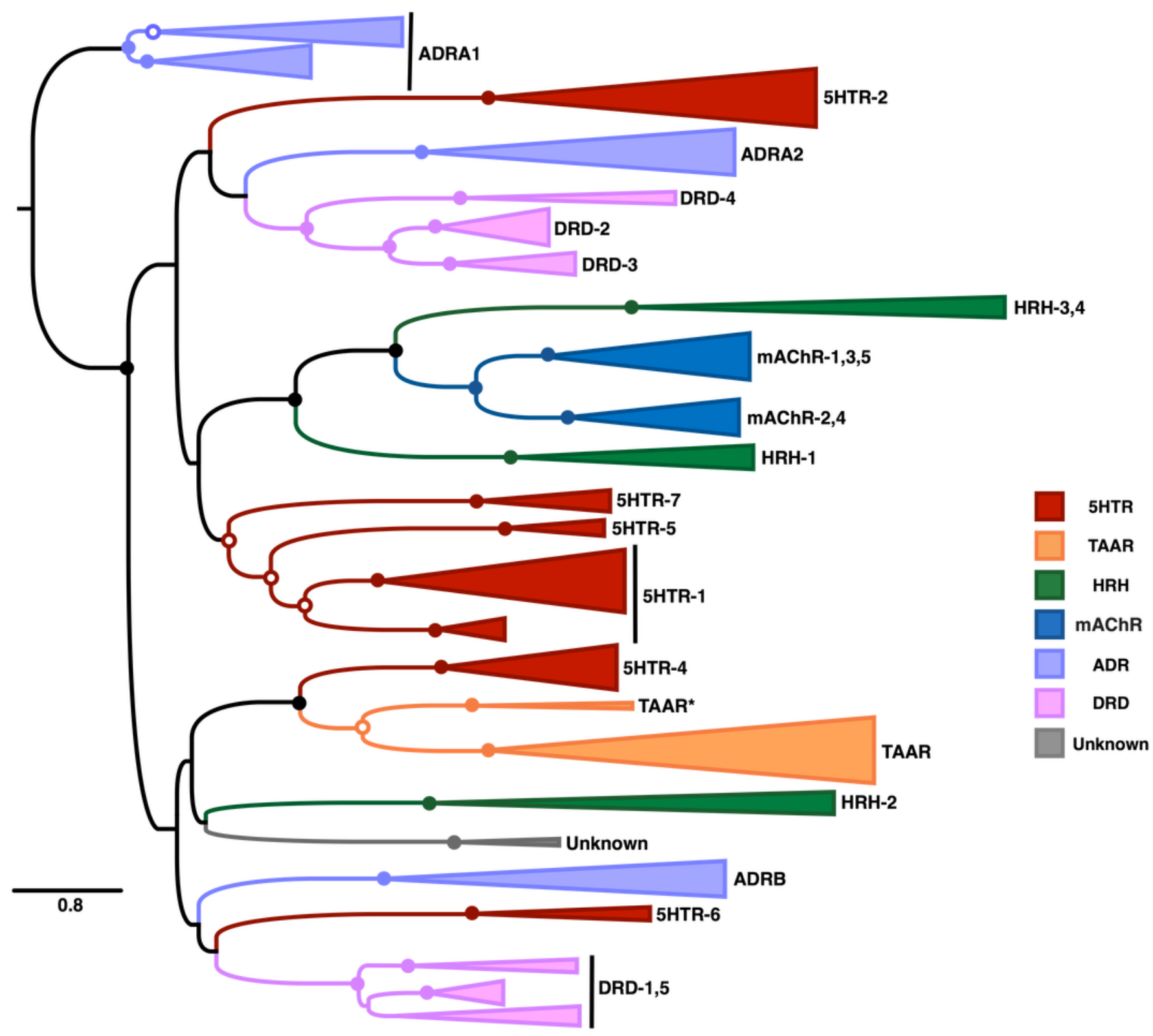




\section{6}

Subclade of the TAAR receptors within the phylogeny shown in Figure 5.

Nodes with open circles indicate $\geq 50 \%$ bootstrap support, and nodes with closed circles indicate $\geq 90 \%$ bootstrap support. The subclades within the TAAR-6,7,8,9 clade labeled as "B" and "R" indicate clades containing only bovid and rodent sequences, respectively.

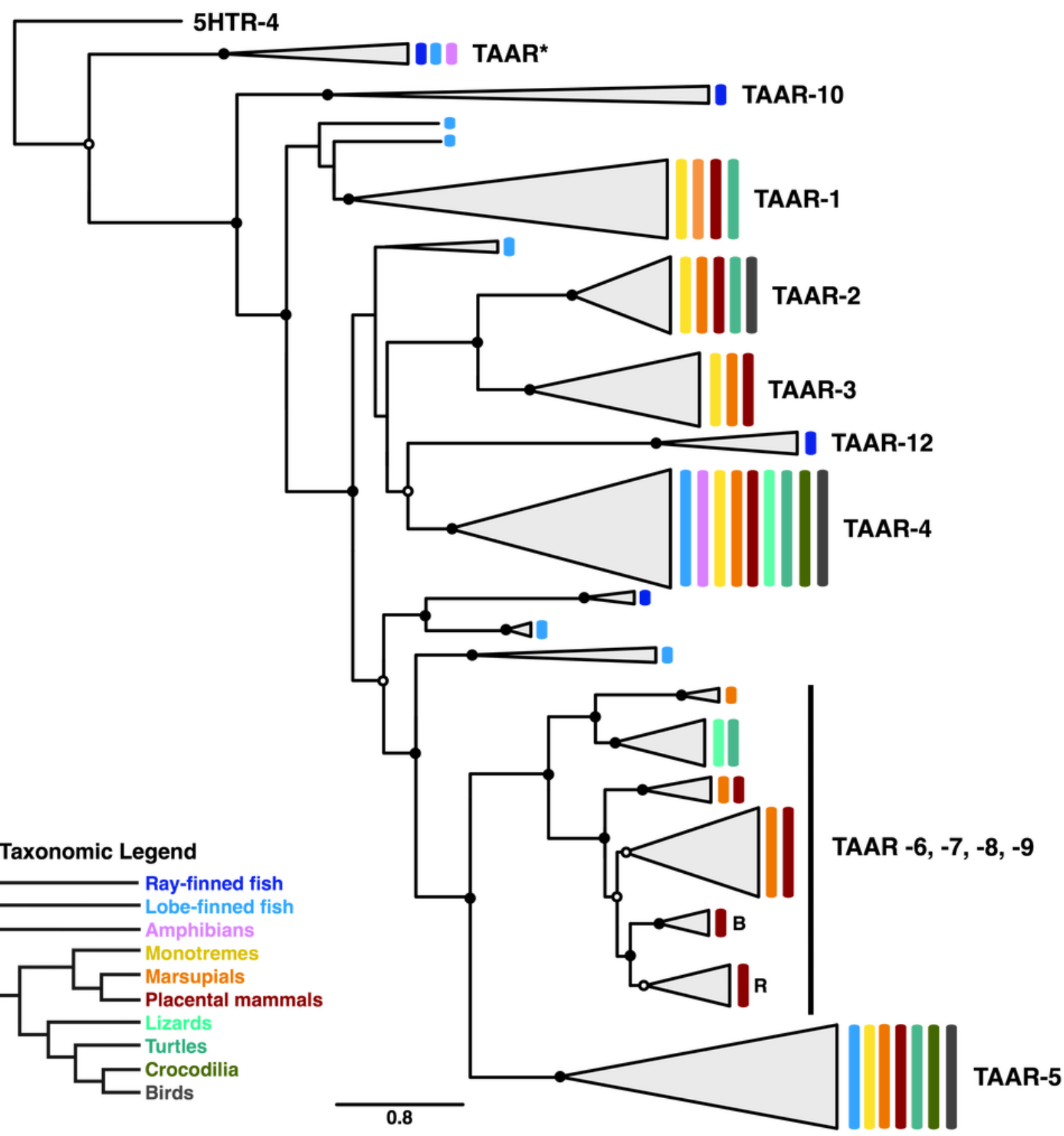

